\title{
Fundamental groups of compact manifolds and symmetric geometry of noncompact type
}

\author{
A. Candel ${ }^{1}$ and R. Quiroga-Barranco ${ }^{2}$
}

\begin{abstract}
We introduce the notion of geometrical engagement for actions of semisimple Lie groups and their lattices as a concept closely related to Zimmer's topological engagement condition. Our notion is a geometrical criterion in the sense that it makes use of Riemannian distances. However, it can be used together with the foliated harmonic map techniques introduced in [8] to establish foliated geometric superrigidity results for both actions and geometric objects. In particular, we improve the applications of the main theorem in [9] to consider nonpositively curved compact manifolds (not necessarily with strictly negative curvature). We also establish topological restrictions for Riemannian manifolds whose universal cover have a suitable symmetric de Rham factor (Theorem B), as well as geometric obstructions for nonpositively curved compact manifolds to have fundamental groups isomorphic to certain groups build out of cocompact lattices in higher rank simple Lie groups (Corollary 4.5).
\end{abstract}

Mathematics Subject Classification (1991). Primary 53C12, 58E20; Secondary 58G11, $28 \mathrm{~A} 33$.

Keywords. Symmetric spaces, semisimple groups, foliations.

\section{Introduction}

One of the main problems in the study of the superrigid properties of symmetric spaces of noncompact type, and their groups of isometries, is to determine the restrictions on the fundamental groups of manifolds whose geometry and/or dynamics are closely related to such spaces. In this direction, we found results by Ballmann-Eberlein [2], Gromov [3] and Spatzier-Zimmer [9] which show (among other things) that a compact manifold whose geometry (dynamics in the case of [9]) is that of a rank at least 2 irreducible symmetric space of noncompact type cannot have the fundamental group of a compact manifold with strictly negative curvature.

\footnotetext{
${ }^{1}$ Research supported by N.S.F. Grant No. INT-9600468.

${ }^{2}$ Research supported by SNI-MEXICO, CINVESTAV-IPN-MEXICO and grants CONACYT E120.3148 and JIRA-97/043.
} 
On the other hand, by using the foliated heat flow theory developed by Gromov [5], it has been possible to extend geometric superrigidity techniques to compact foliated manifolds as found in [1] and [8]. These works provide restrictions on possible leafwise geometries of compact foliated manifolds which already carry a leafwise symmetric geometry; in [8] it is further developed a way to compare the geometry of such compact foliated manifolds to that of a compact nonpositively curved manifold. However, the following remarks point to some problems not considered in these works: 1) The results from [1] and [8] make use of diffeomorphisms as a mean of comparison between manifolds and, even though they impose some useful geometric restrictions, they are not truly showing topological restrictions. 2) The notion of stretch of a foliation introduced by Gromov and used in [8] to solve foliated geometric superrigidity problems has a geometric/dynamic nature, which was not further studied in [8], and this feature should be the key step in extending the techniques of [8] from foliations to suitable actions of semisimple Lie groups.

The main contribution of this work is to deal with the above remarks by providing a geometric notion of Zimmer's topological engagement condition which is then used, together with the foliated techniques from [8], to prove rigidity results (in the sense of providing topological obstructions) for compact manifolds with suitable geometry or dynamics closely related to that of certain symmetric spaces of noncompact type.

One of the main tools used in [8], as well as in this work, is the notion of a positively stretched foliation which is spelled out in section 2 . To better understand the techniques and main results of this work let us just say at this point that to a compact manifold $M$ carrying a foliation $\mathcal{F}$, a leafwise Riemannian metric $g$ and a finite invariant transverse measure one associates a nonnegative real number $\operatorname{stre}(M, \mathcal{F}, g)$ which provides a geometric measure of the properness of the foliation when lifted to the universal cover; if $(M, \mathcal{F}, g)$ is positively stretched, i.e. $\operatorname{stre}(M, \mathcal{F}, g)>0$, then, in some sense, there is a non-null collection of geometrically proper leaves in the universal cover of $M$. We take a step further and say that suitable actions of semisimple Lie groups are geometrically engaging if, when lifted to the universal cover of the manifold being acted upon, the orbits are proper in the sense of a Riemannian distance. Such restriction is similar to the topological engagement condition considered by Zimmer in [9].

The main results are the following:

Theorem A. Let $M$ be a manifold with a smooth foliation $\mathcal{F}$ carrying a finite invariant transverse measure $\mu$ and a leafwise Riemannian metric $g$. Assume that each leaf is isometrically covered by a fixed irreducible symmetric space $X$ of noncompact type which is either of rank at least 2 or a quaternionic or Cayley hyperbolic space, and that $\operatorname{stre}(M, \mathcal{F}, g)>0$. If $N$ is a compact manifold with nonpositive sectional curvature when $\operatorname{rank}(X) \geq 2$ and with nonpositive complexified sectional curvature otherwise and $\pi_{1}(M) \cong \pi_{1}(N)$, then there exist a homothetic 
totally geodesic immersion $X \rightarrow N$.

This theorem improves one of the main results from [8] by obtaining a geometric restriction (the totally geodesic immersion $X \rightarrow N$ ) from just an isomorphism of fundamental groups. In [8], to obtain a similar conclusion, it was required either a diffeomorphism between $M$ and $N$ or a smooth map $M \rightarrow N$ with certain geometric/dynamical restrictions.

Theorem B. Let $(M, g)$ be a compact Riemannian manifold whose universal cover can be isometrically split as $\widetilde{M} \cong Y \times X$, where $X$ is as in Theorem A. Let $N$ be a compact manifold with nonpositive sectional curvature when $\operatorname{rank}(X) \geq 2$ and with nonpositive complexified sectional curvature otherwise. If $\pi_{1}(M) \cong \pi_{1}(N)$, then there is a homothetic totally geodesic immersion $X \rightarrow N$. In particular, for $\operatorname{rank}(X) \geq 2$ the group $\pi_{1}(M)$ cannot be isomorphic to the fundamental group of a compact manifold with strictly negative sectional curvature.

This last result can be rephrased by saying that if a nonpositively curved compact manifold $N$ has the same fundamental group of a compact manifold $M$ whose geometry is only "partially" symmetric of rank at least 2 , then $N$ contains some of that geometry immersed in a homothetic and totally geodesic fashion, and so it can be considered itself as "partially" symmetric. Theorem B' from section 5 states a similar result for Riemannian foliations.

As for results involving the dynamics of the group of isometries of a symmetric space we prove the following:

Theorem C. Let $X$ and $N$ be as in Theorem A, and let $\Gamma$ be a torsion free cocompact lattice of the group of isometries of $X$. Assume $\Gamma$ has a geometrically engaging action on a compact manifold $T$ that preserves a finite measure. If $\pi_{1}(T) \cong \pi_{1}(N)$, then there is an isometric totally geodesic immersion $X \rightarrow N$. In particular, when $\operatorname{rank}(X) \geq 2$, the space $T$ cannot have the fundamental group of a compact manifold with strictly negative sectional curvature.

This result is similar to the main theorem found in [9], where the geometrical engagement condition replaces the assumption on the existence of an invariant connection used in [9]. We remark that some of the arguments found in [8] prove that a geometrically engaging action is topologically engaging and, since the latter is the chief tool used to prove the main result in [9], it follows that the particular case stated in Theorem $\mathrm{C}$ is a consequence of [9]. However, Theorem $\mathrm{C}$ imposes obstructions given the condition $\pi_{1}(T) \cong \pi_{1}(N)$ even if $N$ does not necessarily have strictly negative sectional curvature, which is an essential hypothesis in the arguments found in [9] when using topological engagement. On the other hand, for higher rank groups as above the known measure preserving actions are of an algebraic nature or obtained by gluing such algebraic constructions (see 4 and 
section 4), and we prove that they all are geometrically engaging (see Theorems 3.3 and 3.4), so the condition is not very restrictive. Also, our proofs use only geometry and harmonic map theory, so that the particular case of Theorem $\mathrm{C}$ provides a geometric proof (in fact, a geometric superrigidity proof) of the main theorem from [9] for all known measure preserving actions.

As a matter of fact, the geometric engagement of some "exotic" actions (as described in section 4) is used to establish an obstruction for nonpositively curved compact manifolds to have certain fundamental groups build out of lattices. More precisely, let $H$ be a simply connected simple Lie group and $G$ a simple subgroup, both of noncompact type and rank at least 2. Assume that $G$ has codimension at least 3 in $H$ and that there is a torsion free cocompact lattice $\Lambda$ of $H$ such that $\Gamma=\Lambda \cap G$ is a lattice in $G$. Then we have the following:

Corollary 4.5. Let $N$ be a compact manifold with nonpositive sectional curvature. If $\pi_{1}(N)$ is isomorphic to the amalgamated product $\Lambda *_{\Gamma} \Lambda$ or to the $H N N-$ extension $\Lambda *_{\Gamma}$ (both constructed from the identity isomorphism of $\Gamma$ ), then there is an isometric totally geodesic immersion of the symmetric space associated to $G$ in $N$. In particular, the fundamental group of a compact manifold with strictly negative sectional curvature cannot be isomorphic to either $\Lambda *_{\Gamma} \Lambda$ or $\Lambda *_{\Gamma}$.

\section{Preliminaries}

In this section we recall some results and definitions from [8] related to the notion of stretch and foliated geometric superrigidity. We also define geometric engagement as a dynamical version of the property of having positive stretch. Also some auxiliary results are stated and/or proved in this section.

Definition 2.1. Let $M$ be a compact metric space with a smooth foliation $\mathcal{F}$ carrying a smooth leafwise Riemannian metric $g,(N, h)$ a compact Riemannian manifold and $f: M \rightarrow N$ a smooth map. For $v$ in $U T_{\mathcal{F}} M$ (the leafwise unit tangent bundle of $M$ ) let $\gamma$ be the $g$-geodesic with initial velocity vector $v$ lying within the leaf $\mathcal{L}$ to which $v$ is tangent, let $\widetilde{\gamma}$ be any lift of $\gamma$ to the universal cover of $\mathcal{L}$ and $\widetilde{f}(\widetilde{\gamma})$ its image under a lift $\widetilde{f}$ to universal covers of the restriction $\left.f\right|_{\mathcal{L}}$. Define:

$$
\phi_{t}(v)=d_{(\widetilde{N}, h)}(\widetilde{f}(\widetilde{\gamma}(0)), \widetilde{f}(\widetilde{\gamma}(t)))
$$

Then the pointwise stretch of $f$ is the function defined by:

$$
\begin{gathered}
\text { p-stre }(f): U T_{\mathcal{F}} M \rightarrow \mathbb{R} \\
v \mapsto \liminf _{t \rightarrow \infty} \frac{\phi_{t}(v)}{t}
\end{gathered}
$$


If $\mathcal{F}$ carries a finite invariant transverse measure $\mu$, the stretch of $f$ is defined by:

$$
\operatorname{stre}(f)=\lim _{t \rightarrow \infty} \int_{U T_{\mathcal{F}} M} \frac{\phi_{t}(v)}{t} d \mu_{L}=\int_{U T_{\mathcal{F}} M} \mathrm{p} \text {-stre }(f) d \mu_{L}
$$

where $\mu_{L}$ is the measure on $U T_{\mathcal{F}} M$ obtained by integrating against $\mu$ the Riemannian measures on the leaves of the foliation in $U T_{\mathcal{F}} M$ induced by $\mathcal{F}$.

Remark 2.2. It is an easy matter to verify the last identity on the previous definition.

The pointwise stretch of a function $f$ is nonnegative, and we will say that $f$ has positive pointwise stretch if it is a positive function; observe that this condition does not depend on the choice of the metric on $N$. The same kind of remarks apply to the stretch.

For the particular case $M=N$ and $f=i d_{M}$, the identity map on $M$, the above are called the pointwise stretch and the stretch of $(M, \mathcal{F}, g)$ and are denoted by p-stre $(M, \mathcal{F}, g)$ and $\operatorname{stre}(M, \mathcal{F}, g)$, respectively.

Remark 2.3. The notion of a positive stretch map, being formally similar to that of quasi-isometry, is not equivalent to it. An example that illustrates this is the following. Take two Reeb foliations of a strip in the plane, glue them together to obtain a foliation of a cylinder, then mod out by a translation to get a foliation of the flat two-dimensional torus. In the universal cover of the torus we have a foliation whose leaves can be assumed to be the translates of the graphs of the curves $y=\exp \left(\left(1-x^{2}\right)^{-2}\right)$ and vertical lines $x= \pm 1$. It takes a short calculation to verify that each graph has positive stretch but, excepting the lines, is not quasiisometrically embedded.

Similar examples can be constructed in hyperbolic space, simply by taking two geodesics with one common endpoint at infinity, and then inserting in between them a Reeb-like foliation.

Definition 2.4. Let $G$ be a connected Lie group locally isomorphic to the group of isometries of a symmetric space $X$ of noncompact type and let $T$ be a compact Riemannian manifold acted upon by $G$. Choose a Cartan decomposition $\mathfrak{g}=\mathfrak{k} \oplus \mathfrak{m}$ for the Lie algebra of $G$ (with $\mathfrak{k}$ a maximal compact subalgebra), and let $\mathfrak{m}_{1}$ be the unit ball in $\mathfrak{m}$ with respect to the Killing form of $\mathfrak{g}$. For $v \in \mathfrak{m}_{1}$ denote with $g_{t}^{v}=\exp (t v)$ the one-parameter subgroup of $\widetilde{G}$ generated by $v$. The pointwise stretch of the action of $G$ on $T$ is the function defined by:

$$
\begin{aligned}
& \text { p-stre }(G, T): \mathfrak{m}_{1} \times \widetilde{T} \rightarrow \mathbb{R} \\
& (v, x) \mapsto \liminf _{t \rightarrow \infty} \frac{d_{\widetilde{T}}\left(g_{t}^{v} x, x\right)}{t}
\end{aligned}
$$

where $\widetilde{G}$ acts on $\widetilde{T}$ by an arbitrary but fixed lift of the action of $G$ on $T$. We say that the action of $G$ on $T$ has positive stretch if p-stre $(G, T)$ is a positive function. 
Remark 2.5. Observe that the pointwise stretch of an action does not depend on the choice of the lift of the action of $G$ on $T$ to an action of $\widetilde{G}$ on $\widetilde{T}$. On the other hand, the pointwise stretch of an action does depend on the choice of the Cartan decomposition and the Riemannian metric on $T$. However, the property of having positive stretch does not depend on any of them. Also notice that the action of $G$ on $T$ has positive stretch if and only if for every one-parameter subgroup $g_{t}$ of $\widetilde{G}$ which does not map into a compact subgroup of $\operatorname{Ad}(G)$ we have:

$$
\liminf _{t \rightarrow \infty} \frac{d_{\widetilde{T}}\left(g_{t} x, x\right)}{t}>0
$$

for every $x \in \widetilde{T}$. From this it is a straightforward matter to check that an action with positive stretch is topologically engaging.

Since the orbit under $g_{t}$ (as in the definition above) of a suitable point in $X$ is a geodesic, with $t$ proportional to the length parameter, we can say that the action of $G$ on $T$ has positive stretch if the action lifted to universal covers is such that the orbits of "noncompact" one-parameter subgroups increase their length at least as fast as they do within $X$. Hence, a positive stretch action has geometric/dynamical properties similar to those of the action of $\widetilde{G}$ on $X$.

Definition 2.6. With the same notation as in the previous definition, we say that the action $G \times T \rightarrow T$ of the Lie group $G$ on the manifold $T$ has uniform positive stretch if:

$$
\liminf _{t \rightarrow \infty} \inf _{v \in \mathfrak{m}_{1}} \frac{d_{\widetilde{T}}\left(g_{t}^{v} x, x\right)}{t}>0
$$

for every $x \in \widetilde{T}$.

In words, an action with uniform positive stretch is one that has at every point positive stretch bounded from below by a fixed constant for all directions of the symmetric space associated to $G$. A property like this would be good enough to provide the conclusions of our theorems, but we actually need less. We introduce the notion of geometrically engaging action; this concept implies positive stretch, and it is implied by uniform positive stretch. It is close in spirit to the notion of topologically engaging action discussed in [9].

Definition 2.7. With the notation as in the previous definitions, we say that the action of $G$ on $T$ is geometrically engaging if for every sequence $\left(g_{n}\right)_{n}$ in $G$ such that $\left(g_{n} x_{0}\right)_{n}$ is a quasi-ray in $X$ (the symmetric space associated to $G$ ) for some (and hence any) $x_{0} \in X$, the limit inferior:

$$
\liminf _{n \rightarrow \infty} \frac{d_{\widetilde{T}}\left(g_{n} x, x\right)}{d_{X}\left(g_{n} x_{0}, x_{0}\right)}>0
$$

for every $x \in \widetilde{T}$. 
Definition 2.8. Let $\Gamma$ be a cocompact lattice in the group of isometries of a symmetric space $X$ of noncompact type, and let $T$ be a compact manifold. An action of $\Gamma$ on $T$ is called geometrically engaging if it is induced by a $\Gamma$-action on the principal bundle $\widetilde{T} \rightarrow T$ in such a way that for every sequence $\left(\gamma_{n}\right)_{n}$ in $\Gamma$ defining a quasi-ray (for a word metric) we have that for some (and hence any) $x_{0} \in X$, the limit inferior:

$$
\liminf _{n \rightarrow \infty} \frac{d_{\widetilde{T}}\left(\gamma_{n} x, x\right)}{d_{X}\left(\gamma_{n} x_{0}, x_{0}\right)}>0
$$

for every $x \in \widetilde{T}$.

Remark 2.9. Recall that a sequence $x_{n}$ in $X$ is called a quasi-ray if there exist constants $A>0$ and $B \geq 0$ such that

$$
A^{-1}|m-n|-B \leq d_{X}\left(x_{n}, x_{m}\right) \leq A|m-n|+B
$$

for all $m, n \geq 0$. Also recall that the image of a geodesic ray under a quasi-isometry is a quasi-ray.

Remark 2.10. Let $G$ be a simply connected semisimple Lie group of noncompact type and $\Gamma$ a cocompact lattice. If $\left(\gamma_{n}\right)_{n}$ is quasi-ray in $\Gamma$ for some word metric, then $\left(\gamma_{n} x_{0}\right)_{n}$ is a quasi-ray in $X$ (the symmetric space associated to $G$ ) for any $x_{0} \in X$. In particular, any geometrically engaging action of $G$ induces a geometrically engaging action of $\Gamma$.

Remark 2.11. It is clear that the notion of geometrically engaging action is independent of the point $x_{0}$ of $X$ because $G$ is the isometry group of $X$. Also note that the actual value of the limit inferior may depend on the point $x$ in $T$.

The concept of geometric engagement ensures that the geometric/dynamical properties of the action of $\widetilde{G}$ on $\widetilde{T}$ are close to those of the action of $\widetilde{G}$ on $X$, but now from the point of view of orbits by sequences in $\widetilde{G}$ which are only nearby to the one-parameter subgroups considered in definition 2.4. It is stronger than topological engagement, which only requires the existence of an element of $G$ (not in a conjugate of the isotropy group of $X$ ) whose orbit in $\widetilde{T}$ is locally closed (see $[9])$.

Now we state some simply facts about cocycles which will be needed later.

Definition 2.12. Let $G$ and $H$ be Lie groups such that the former acts on a Borel measure space $T$. A Borel mapping $\alpha: G \times T \rightarrow H$ is called a cocycle if for every $g, g^{\prime} \in G$ it satisfies:

$$
\alpha\left(g g^{\prime}, x\right)=\alpha\left(g, g^{\prime} x\right) \alpha\left(g^{\prime}, x\right)
$$


for a.e. $x \in T$. The cocycle is called strict whenever this condition is satisfied for every $x \in T$.

Remark 2.13. We recall that if $G$ is Lie group acting by bundle automorphisms on a principal bundle $p: P \rightarrow M$ with structure group $H$, then any Borel section $s$ of $p$ induces a cocycle $\alpha: G \times M \rightarrow H$ which is defined so that it satisfies:

$$
g s(m)=s(g m) \alpha(g, m)
$$

for every $g \in G$ and a.e. $m \in M$. Moreover, if $s$ is a section in the sense of sets, then $\alpha$ can be assumed to be strict.

Lemma 2.14. Let $T$ be a compact Riemannian manifold and $\Gamma$ a cocompact lattice of the group of isometries of a symmetric space of noncompact type. Assume that $\Gamma$ acts on the principal bundle $\widetilde{T} \rightarrow T$. Then there exist a Borel section $s: T \rightarrow \widetilde{T}$ of the universal covering $p: \widetilde{T} \rightarrow T$ which is a bijection onto $s(T)$ and such that the latter is Borel with diameter $\leq 6 \operatorname{diam}(T)$. In particular, the section $s$ induces a strict cocycle $\alpha_{0}: \gamma \times T \rightarrow \pi_{1}(T)$, for the $\Gamma$-action on $\widetilde{T} \rightarrow T$, which satisfies:

$$
d_{\widetilde{T}}\left(\gamma s(x), s(x) \alpha_{0}(\gamma, x)\right) \leq 6 \operatorname{diam}(T)
$$

for any $\gamma \in \Gamma$ and $x \in T$.

Proof. Let $\left\{B_{i}\right\}_{i=1}^{n}$ be a cover of $T$ by evenly covered open balls (i.e. the restricted bundle $\left.\pi\right|_{\pi^{-1}\left(B_{i}\right)}: \pi^{-1}\left(B_{i}\right) \rightarrow B_{i}$ is trivial), and for each $i$ choose a lift $\widetilde{B}_{i}$ of $B_{i}$ such that all of them lie within a fixed ball of radius $3 \operatorname{diam}(T)$. Define $A_{1}=\widetilde{B}_{1}$, $A_{i}=\widetilde{B}_{i} \backslash\left(p^{-1}\left(B_{1} \cup \cdots \cup B_{i-1}\right)\right)$; then $A=\bigcup_{i=1}^{n} A_{i}$ is Borel with diameter $\leq 6 \operatorname{diam}(T)$ such that $\left.p\right|_{A}: A \rightarrow T$ is a bijection, and so the latter defines a Borel map $s=\left.p\right|_{A} ^{-1}$ which is a section of $p$ in the sense of sets.

On the other hand, the cocycle induced by $s$ satisfies:

$$
\gamma s(x)=s(\gamma x) \alpha_{0}(\gamma, x)
$$

for every $\gamma, x$. In particular, $\gamma s(x)$ and $s(x) \alpha_{0}(\gamma, x)$ lie within the set $s(T) \alpha_{0}(\gamma, x)$ which has diameter $\leq 6 \operatorname{diam}(T)$ since $\alpha_{0}(\gamma, x)$ is an isometry.

The following is the main result from [8] on foliated geometric superrigidity.

Proposition 2.15. Let $M$ be a compact manifold with a smooth foliation $\mathcal{F}$ carrying a finite invariant transverse measure $\mu$ and a leafwise Riemannian metric $g$. Assume that each leaf is isometrically covered by a fixed irreducible symmetric space $X$ of noncompact type which is either of rank at least 2 or a quaternionic or Cayley hyperbolic space. Let $N$ be a compact Riemannian manifold with nonpositive sectional curvature when the rank of $X$ is at least 2 and with nonpositive 
complexified sectional curvature otherwise. If $f: M \rightarrow N$ is a smooth map with positive stretch, then there is a homothetic totally geodesic immersion $X \rightarrow N$.

The next result from [8], which will be applied later, asserts that suitable compact manifolds have positive stretch for foliations induced by their de Rham factors.

Proposition 2.16. Let $M$ be a compact Riemannian manifold whose universal cover isometrically splits as $\widetilde{M}=Y \times X$, where $X$ is an irreducible Riemannian manifold without conjugate points, and such that the factor $X$ induces a foliation $\mathcal{F}$ on $M$. Then $(M, \mathcal{F})$ is pointwise positively stretched with respect to the leafwise Riemannian metric induced from that of $X$ and the finite invariant transverse measure induced by the Riemannian measure on $Y$.

In order to build positively stretched maps from cocycles we will make use of the following proposition.

Proposition 2.17. Let $\Gamma$ be a discrete group which acts smoothly on the manifolds $X$ and $T$, where the action on the former is free. Let $\alpha: \Gamma \times T \rightarrow \pi_{1}(N)$ be a strict cocycle, where $N$ is a manifold with contractible universal cover. Then there is a smooth $\left(\alpha, \pi_{1}(N)\right)$-equivariant map $\widetilde{f}: X \times T \rightarrow \widetilde{N}$, which in particular induces a smooth map $f: \Gamma \backslash(X \times T) \rightarrow N$.

Proof. Observe that $\Gamma$ acts on $X \times T$ by $\gamma(x, t)=(\gamma x, \gamma t)$ and similarly, the strict cocycle condition on $\alpha$ ensures that the mapping:

$$
\begin{aligned}
& \Gamma \times X \times T \times \tilde{N} \rightarrow X \times T \times \tilde{N} \\
& \gamma(x, t, n) \mapsto\left(\gamma x, \gamma t, n \alpha(\gamma, t)^{-1}\right)
\end{aligned}
$$

defines an action. Moreover, it is easy to check that the natural projection $\Gamma \backslash(X \times$ $T \times \widetilde{N}) \rightarrow \Gamma \backslash(X \times T)$ defines a fiber bundle with fiber $\widetilde{N}$. The manifold $\widetilde{N}$ being contractible, there is a smooth section $s$ and we can define a map $\widetilde{f}: X \times T \rightarrow \widetilde{N}$ by the condition $s([x, t])=[x, t, \widetilde{f}(x, t)]$. One easily shows that $\widetilde{f}$ is well defined and $\left(\alpha, \pi_{1}(N)\right)$-equivariant, i.e. it satisfies:

$$
\widetilde{f}(\gamma(x, t))=\widetilde{f}(x, t) \alpha(\gamma, t)^{-1}
$$

for every $\gamma \in \Gamma, x \in X$ and $t \in T$. Also, by looking at local trivializations of the bundle one can show that $\widetilde{f}$ is smooth. By $\left(\alpha, \pi_{1}(N)\right)$-equivariance, $\widetilde{f}$ induces a map $f: \Gamma \backslash(X \times T) \rightarrow N$ which makes the diagram

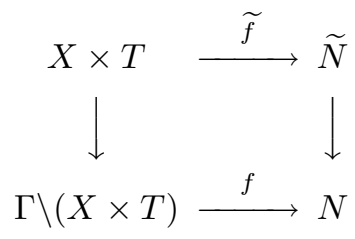


commutative, where the vertical arrows are the natural projections. Notice that the commutativity of the diagram implies the smoothness of $f$.

\section{Fundamental groups and geometric engagement}

In this section we develop some criteria to ensure that suitable maps and foliations are positively stretched, and we also prove that the algebraic measure preserving actions of semisimple Lie groups of noncompact type are geometrically engaging. Our first result considers topological data to get the desired conclusion.

Proposition 3.1. Let $M$ and $N$ be compact manifolds and assume that $M$ carries a smooth foliation $\mathcal{F}$ and a leafwise Riemannian metric $g$ without conjugate points. Let $h$ be a Riemannian metric on $N$. If $f: M \rightarrow N$ is a smooth map which induces on fundamental groups a map $f_{*}: \pi_{1}(M) \rightarrow \pi_{1}(N)$ which is a quasi-isometry, then there is a constant $A>0$ such that:

$$
A \text { p-stre }(M, \mathcal{F}, g) \leq \mathrm{p} \text {-stre }(f)
$$

where p-stre $(f)$ is computed by considering $f$ as a map $f:(M, \mathcal{F}) \rightarrow(N, h)$ for some arbitrary but fixed Riemannian metric $h$ on $N$.

Proof. Choose any global Riemannian metric $s$ over $M$. Since $f_{*}$ is a quasiisometry, it follows that the lift $\widetilde{f}: \widetilde{M} \rightarrow \widetilde{N}$ of the map $f$ to universal covers is a quasi-isometry. Hence, there is a map $\widetilde{g}: \widetilde{N} \rightarrow \widetilde{M}$ and constants $\lambda, C>0$ such that for any $x, y \in \widetilde{M}$ :

$$
\begin{aligned}
& d_{(\widetilde{M}, s)}(\widetilde{g}(x), \widetilde{g}(y)) \leq \lambda d_{(\widetilde{N}, h)}(x, y)+C \\
& d_{(\widetilde{M}, s)}(\widetilde{g}(\widetilde{f}(x)), x) \leq C
\end{aligned}
$$

Let $v \in U T_{\mathcal{F}}(M)$ be given and denote by $\gamma$ the $g$-geodesic in the leaf that contains the basepoint of $v$ and with initial condition $\gamma^{\prime}(0)=v$. Let $\widetilde{\gamma}$ be some lift of $\gamma$ to the universal cover $\widetilde{M}$, and for every $t>0$ define:

$$
\begin{aligned}
& \psi_{t}(v)=d_{(\widetilde{M}, s)}(\widetilde{\gamma}(0), \widetilde{\gamma}(t)) \\
& \phi_{t}(v)=d_{(\widetilde{N}, h)}(\widetilde{f}(\widetilde{\gamma}(0)), \widetilde{f}(\widetilde{\gamma}(t)))
\end{aligned}
$$

In particular, we have:

$$
\begin{aligned}
\operatorname{p}-\operatorname{stre}(M, \mathcal{F}, g)(v) & =\liminf _{t \rightarrow \infty} \frac{\psi_{t}(v)}{t} \\
\operatorname{p}-\operatorname{stre}(f)(v) & =\liminf _{t \rightarrow \infty} \frac{\phi_{t}(v)}{t}
\end{aligned}
$$


Then from inequality (1) it follows that:

$$
\begin{aligned}
d_{(\widetilde{M}, s)}(\widetilde{g}(\tilde{f}(\widetilde{\gamma}(0))), \widetilde{g}(\widetilde{f}(\widetilde{\gamma}(t)))) & \leq \lambda d_{(\widetilde{N}, h)}(\widetilde{f}(\widetilde{\gamma}(0)), \widetilde{f}(\widetilde{\gamma}(t)))+C \\
& =\lambda \phi_{t}(v)+C
\end{aligned}
$$

which together with inequality (2) yields:

$$
\begin{aligned}
\psi_{t}(v) & \leq d_{(\widetilde{M}, s)}(\widetilde{\gamma}(0), \widetilde{g}(\widetilde{f}(\widetilde{\gamma}(0)))) \\
& +d_{(\widetilde{M}, s)}(\widetilde{\gamma}(t), \widetilde{g}(\widetilde{f}(\widetilde{\gamma}(t)))) \\
& +d_{(\widetilde{M}, s)}(\widetilde{g}(\widetilde{f}(\widetilde{\gamma}(0))), \widetilde{g}(\widetilde{f}(\widetilde{\gamma}(t)))) \\
& \leq 3 C+\lambda \phi_{t}(v)
\end{aligned}
$$

After dividing by $t$ and taking the limit inferior as $t \rightarrow \infty$ we obtain:

$$
\frac{1}{\lambda} \mathrm{p} \text {-stre }(M, \mathcal{F}, g) \leq \mathrm{p}-\operatorname{stre}(f)
$$

As an immediate consequence we obtain the following:

Corollary 3.2. Let $M$ and $N$ be as in the Proposition 3.1 and let $f: M \rightarrow N$ be a smooth map. If $(M, \mathcal{F}, g)$ is pointwise positively stretched and $f$ induces an isomorphism $f_{*}: \pi_{1}(M) \rightarrow \pi_{1}(N)$, then $f$ is pointwise positively stretched.

The following result shows that the algebraic actions of semisimple Lie groups are geometrically engaging.

Theorem 3.3. Let $G$ be a closed subgroup of a Lie group $H$, both assumed to be semisimple of noncompact type, and $\Lambda$ a cocompact lattice in $H$. Then the action of $G$ on $H / \Lambda$ is geometrically engaging. In particular, the action on $H / \Lambda$ of any cocompact lattice of $\widetilde{G}$ is geometrically engaging.

Proof. Denote with $\mathfrak{g}$ and $\mathfrak{h}$ the Lie algebras of $G$ and $H$, respectively. Let $\mathfrak{g}=\mathfrak{k} \oplus \mathfrak{m}$ and $\mathfrak{h}=\mathfrak{l} \oplus \mathfrak{n}$ be Cartan decompositions with $\mathfrak{k}$ and $\mathfrak{l}$ compact subalgebras. Since $\mathfrak{g}$ can be canonically embedded into $\mathfrak{h}$ (i.e. a Cartan decomposition on $\mathfrak{h}$ is chosen so that its involution leaves $\mathfrak{g}$ invariant, see [7] for more details) we can assume that $\mathfrak{k} \subset \mathfrak{l}$ and $\mathfrak{m} \subset \mathfrak{n}$. Choose a right $H$-invariant $A d_{H}(L)$-invariant (and hence left $L$-invariant) Riemannian metric $h$ on $H$ such that $\mathfrak{l} \perp \mathfrak{n}$ and whose restriction to $\mathfrak{n}$ coincides with the Killing form of $\mathfrak{h}$. The metric $h$ induces a Riemannian metric on $H / \Lambda$, and we will prove that for this choice the action of $G$ on $H / \Lambda$ is geometrically engaging. 
Let $\widetilde{H}$ be the universal cover of $H$ and $\widehat{G}$ the closed connected subgroup of $\widetilde{H}$ with Lie algebra $\mathfrak{g}$. Since the metric on $\widetilde{H}$ is right $\widetilde{H}$-invariant and the right action of $\widetilde{H}$ on itself is equivariant with respect to the left $\widehat{G}$-action, it is enough to check geometric engagement at the identity $e$.

Let $\widetilde{L}$ and $\widehat{K}$ be the closed subgroups of $\widetilde{H}$ with Lie algebras $\mathfrak{l}$ and $\mathfrak{k}$, respectively. Then the metric on $\widetilde{H}$ induces a Riemannian metric on $\widetilde{L} \backslash \widetilde{H}$ such that the natural projection $\pi: \widetilde{H} \rightarrow \widetilde{L} \backslash \widetilde{H}$ is a Riemannian submersion. Also, observe that the metric on $\widetilde{L} \backslash \widetilde{H}$ is $\widetilde{H}$-invariant and so it is isometric to the symmetric space associated to $H$. Moreover, the natural inclusion $\widehat{K} \backslash \widehat{G} \hookrightarrow \widetilde{L} \backslash \widetilde{H}$ realizes the symmetric space $X=\widehat{K} \backslash \widehat{G}$ associated with $G$ as a totally geodesic Riemannian submanifold of $\widetilde{L} \backslash \widetilde{H}$.

Now let $\left(g_{n}\right)_{n}$ be a sequence in $\widehat{G}$ such that $\left(\widehat{K} g_{n}\right)_{n}$ is a quasi-ray in the symmetric space $X=\widehat{K} \backslash \widehat{G}$. For each $n$, let $c_{n}$ be the geodesic segment in $\widetilde{H}$ from $e$ to $g_{n}$ such that length $\left(c_{n}\right)=d_{(\widetilde{H}, h)}\left(g_{n}, e\right)$. Since $\pi$ is a Riemannian submersion and $\pi \circ c_{n}$ has $\widetilde{L} e$ and $\widetilde{L} g_{n}$ as endpoints it follows that:

$$
\operatorname{length}\left(c_{n}\right) \geq \operatorname{length}\left(\pi \circ c_{n}\right) \geq d_{\widetilde{L} \backslash \widetilde{H}}\left(\widetilde{L} g_{n}, \widetilde{L} e\right)=d_{\widehat{K} \backslash \widehat{G}}\left(\widetilde{L} g_{n}, \widetilde{L} e\right)
$$

where the last identity follows from the fact that $\widetilde{L} e$ and $\widetilde{L} g_{n}$ lie in $\widehat{K} \backslash \widehat{G}$ which is a totally geodesic submanifold of $\widetilde{L} \backslash \widetilde{H}$. In particular, we have:

$$
\liminf _{n \rightarrow \infty} \frac{d_{(\widetilde{H}, h)}\left(g_{n}, e\right)}{d_{X}\left(x_{0} g_{n}, x_{0}\right)} \geq 1
$$

for $X=\widehat{K} \backslash \widehat{G}$ the realization given above of the symmetric space associated to $G$ and $x_{0}=\widehat{K}$ the class of the identity. In particular, the action under consideration is geometrically engaging at $e$ and hence at every point.

Remark 3.4. In the proof above we have considered a realization of the symmetric space associated to $G$ by a quotient of the type $K \backslash G$. However, the usual realization of the symmetric space $X$ associated to $G$ (as used in the definition of geometric engagement) is given by $X=G / K$. This causes no conflict since they are isometric under the map $K g \mapsto g^{-1} K$.

The following result is the key step to prove Theorem C:

Proposition 3.5. Let $\Gamma$ a torsion free cocompact lattice of the group of isometries of a symmetric space $X$ of noncompact type with $\Gamma$ acting on the left on $X, N$ a compact manifold with contractible universal cover and $T$ a compact manifold on which $\Gamma$ acts smoothly preserving a finite measure $\mu$. Assume that $\pi_{1}(T) \cong \pi_{1}(N)$ and that the action of $\Gamma$ on $T$ is geometrically engaging. Let $\Gamma$ act diagonally on $X \times T$ and endow $\Gamma \backslash(X \times T)$ with the obvious foliation and leafwise Riemannian 
metric coming from the symmetric space $X$. Then there is a smooth map $f: \Gamma \backslash(X \times$ $T) \rightarrow N$ such that, for any Riemannian metric on $N$, the map $f$ has positive stretch.

Proof. Let $\alpha_{0}: \Gamma \times T \rightarrow \pi_{1}(T)$ be a Borel cocycle that satisfies the conditions from Lemma 2.14. The cocycle $\alpha_{0}$ composed with an isomorphism $\rho: \pi_{1}(T) \rightarrow \pi_{1}(N)$ (which we fix from now on) yields a strict cocycle $\alpha: \Gamma \times T \rightarrow \pi_{1}(N)$, and we consider the maps $\tilde{f}$ and $f$ provided by Proposition 2.17 .

Let $h$ be a Riemannian metric on $N$ and denote with $g$ the Riemannian metrics on both $X$ and $\Gamma \backslash(X \times T)$, the latter being leafwise; also observe that the $\Gamma-$ invariant finite measure $\mu$ on $T$ induces a finite invariant transverse measure on $\Gamma \backslash(X \times T)$. To show that $f$ has positive stretch, the Riemannian metric we put on $\Gamma \backslash(X \times T)$ is such that its restriction to the leaves coincides with the metric $g$ on $X$, and makes the transversals $T$ orthogonal to the leaves. This metric is obviously $\Gamma$-invariant when lifted to $X \times T$.

Choose $\left(x_{0}, t_{0}\right) \in X \times T$ and $v \in T_{x_{0}} X$ unitary, and let $g_{t}$ be a one-parameter subgroup of $G$ (the group of isometries of $X$ ) such that $t \mapsto g_{t} x_{0}$ is the geodesic in $X$ with initial velocity vector $v$. Then the curve defined by $c(t)=\left[g_{t} x_{0}, t_{0}\right]$ is a leafwise geodesic in $\Gamma \backslash(X \times T)$, and the pointwise stretch of $f$ at $\left[x_{0}, t_{0}\right]$ in the direction of the vector $v$ is given by:

$$
\text { p-stre }(f)(v)=\liminf _{t \rightarrow \infty} \frac{d_{\widetilde{N}}\left(\widetilde{f}\left(g_{t} x_{0}, t_{0}\right), \widetilde{f}\left(x_{0}, t_{0}\right)\right)}{t}
$$

Observe that for any $g, \gamma \in G$ we have, for some constant $A>0$,

$$
\begin{aligned}
\left|d_{\widetilde{N}}(\widetilde{f}(x, t), \widetilde{f}(g x, t))-d_{\widetilde{N}}(\widetilde{f}(x, t), \widetilde{f}(\gamma x, t))\right| & \leq d_{\widetilde{N}}(\widetilde{f}(g x, t), \widetilde{f}(\gamma x, t)) \\
& \leq A d_{X \times T}((g x, t),(\gamma x, t)) \\
& =A d_{X}(g x, \gamma x),
\end{aligned}
$$

for all $(x, t) \in X \times T$, where the second inequality follows from the fact that $\tilde{f}$ descends to the map $f$, which is a smooth map between compact manifolds (hence Lipschitz), and the last equality because of the choice of metric in $\Gamma \backslash(X \times T)$.

Let $\mathfrak{g}=\mathfrak{k} \oplus \mathfrak{m}$ be the Cartan decomposition of the Lie algebra of $G$, where $\mathfrak{k}$ is the Lie algebra of the isotropy group of $x_{0}$. Now choose a mapping $F: \exp (\mathfrak{m}) \rightarrow \Gamma$ obtained as the composition of the natural homeomorphism $\exp (\mathfrak{m}) \rightarrow X$ and a quasi-isometry $X \rightarrow \Gamma$. One can further assume that:

$$
d_{X}\left(g x_{0}, F(g) x_{0}\right) \leq 2 \operatorname{diam}(\Gamma \backslash X)
$$

for all $x \in X$ and $g \in \exp (\mathfrak{m})$. From equations (3) and (4) it follows that:

$$
\text { p-stre }(f)(v)=\liminf _{t \rightarrow \infty} \frac{d_{\widetilde{N}}\left(\widetilde{f}\left(x_{0}, t_{0}\right), \widetilde{f}\left(F\left(g_{t}\right) x_{0}, t_{0}\right)\right)}{t}
$$


In order to estimate the last ratio, we first note that since $\widetilde{f}$ is $\left(\alpha, \pi_{1}(N)\right)-$ equivariant we have that

$$
\begin{aligned}
d_{\widetilde{N}}\left(\widetilde{f}\left(x_{0}, t_{0}\right), \widetilde{f}\left(\gamma x_{0}, t_{0}\right)\right) \\
\quad \geq d_{\widetilde{N}}\left(\widetilde{f}\left(x_{0}, t_{0}\right), \widetilde{f}\left(\gamma x_{0}, \gamma t_{0}\right)\right)-d_{\widetilde{N}}\left(\widetilde{f}\left(\gamma x_{0}, \gamma t_{0}\right), \widetilde{f}\left(\gamma x_{0}, t_{0}\right)\right) \\
\quad=d_{\widetilde{N}}\left(\widetilde{f}\left(x_{0}, t_{0}\right), \widetilde{f}\left(x_{0}, t_{0}\right) \alpha\left(\gamma, t_{0}\right)^{-1}\right)-d_{\widetilde{N}}\left(\widetilde{f}\left(\gamma x_{0}, \gamma t_{0}\right), \widetilde{f}\left(\gamma x_{0}, t_{0}\right)\right)
\end{aligned}
$$

for every $\gamma \in \Gamma$.

On the other hand, since for any $y_{0} \in \tilde{N}$ the map $\gamma \mapsto y_{0} \gamma$ defines a quasiisometry $\pi_{1}(N) \rightarrow \widetilde{N}$, it follows that for $d^{\prime}$ a group distance on $\pi_{1}(N)$ there exist constants $\lambda$ and $C$ such that we have:

$$
d^{\prime}\left(e, \alpha\left(\gamma, t_{0}\right)\right) \leq \lambda d_{\widetilde{N}}\left(\widetilde{f}\left(x_{0}, t_{0}\right), \widetilde{f}\left(x_{0}, t_{0}\right) \alpha\left(\gamma, t_{0}\right)^{-1}\right)+C
$$

for all $\gamma \in \Gamma$. Moreover, by the second inequality in (4), the $\Gamma$-invariance of the metric on $X \times T$ and the compactness of $T$ there is a constant $C^{\prime}$ depending only on $x_{0}$ such that:

$$
\begin{aligned}
d_{\widetilde{N}}\left(\widetilde{f}\left(\gamma x_{0}, \gamma t_{0}\right), \widetilde{f}\left(\gamma x_{0}, t_{0}\right)\right) & \leq A d_{X \times T}\left(\left(\gamma x_{0}, \gamma t_{0}\right),\left(\gamma x_{0}, t_{0}\right)\right) \\
& =A d_{X \times T}\left(\left(x_{0}, t_{0}\right),\left(x_{0}, \gamma^{-1} t_{0}\right)\right) \\
& \leq C^{\prime}
\end{aligned}
$$

for every $\gamma \in \Gamma$.

For a suitable choice of generators we can assume that $\rho$ defines an isometry between $\pi_{1}(T)$ and $\pi_{1}(N)$ with their group metrics, so that if we denote with the same symbol $d^{\prime}$ the metric on $\pi_{1}(T)$, then the last three sets of inequalities imply that:

$$
d_{\widetilde{N}}\left(\widetilde{f}\left(x_{0}, t_{0}\right), \widetilde{f}\left(\gamma x_{0}, t_{0}\right) \geq \frac{1}{\lambda} d^{\prime}\left(e, \alpha_{0}\left(\gamma, t_{0}\right)\right)-\frac{C}{\lambda}-C^{\prime}\right.
$$

for every $\gamma \in \Gamma$.

Choose a Riemannian metric on $T$ and let $\widetilde{t}_{0} \in \widetilde{T}$ be the image of $t_{0}$ under the Borel section from Lemma 2.14. Then it follows that for every $\gamma \in \Gamma$ :

$$
d_{\widetilde{T}}\left(\gamma \widetilde{t}_{0}, \widetilde{t}_{0} \alpha_{0}\left(\gamma, t_{0}\right)\right) \leq 6 \operatorname{diam}(T)
$$

Also, recall that by compactness of $T$, the mapping $\gamma \mapsto \widetilde{t}_{0} \gamma$ defines a quasiisometry $\pi_{1}(T) \rightarrow \widetilde{T}$, so there exist constants $\lambda_{0}$ and $C_{0}$ such that for every $\gamma \in \Gamma$ :

$$
d_{\widetilde{T}}\left(\widetilde{t}_{0}, \widetilde{t}_{0} \alpha_{0}\left(\gamma, t_{0}\right)\right) \leq \lambda_{0} d^{\prime}\left(e, \alpha_{0}\left(\gamma, t_{0}\right)\right)+C_{0},
$$

and this together with the previous inequality yields

$$
d_{\widetilde{T}}\left(\widetilde{t}_{0}, \gamma \widetilde{t}_{0}\right) \leq \lambda_{0} d^{\prime}\left(e, \alpha_{0}\left(\gamma, t_{0}\right)\right)+C_{0}+6 \operatorname{diam}(T),
$$


for every $\gamma \in \Gamma$.

From the above it follows that:

$$
\begin{aligned}
\operatorname{p-stre}(f)(v) & =\liminf _{t \rightarrow \infty} \frac{d_{\widetilde{N}}\left(\widetilde{f}\left(x_{0}, t_{0}\right), \widetilde{f}\left(F\left(g_{t}\right) x_{0}, t_{0}\right)\right)}{t} & & \text { by }(5) \\
& \geq \frac{1}{\lambda} \liminf _{t \rightarrow \infty} \frac{d^{\prime}\left(e, \alpha_{0}\left(F\left(g_{t}\right), t_{0}\right)\right)}{t} & & \text { by }(6) \\
& \geq \frac{1}{\lambda \lambda_{0}} \liminf _{t \rightarrow \infty} \frac{d_{\widetilde{T}}\left(\widetilde{t_{0}}, F\left(g_{t}\right) \widetilde{t}_{0}\right)}{t} & & \text { by }(7)
\end{aligned}
$$

To see that the last limit inferior above is positive, we first observe that since $F$ is a quasi-isometry:

$$
\lim _{t \rightarrow \infty} \frac{d_{X}\left(x_{0}, F\left(g_{t}\right) x_{0}\right)}{d_{X}\left(x_{0}, g_{t} x_{0}\right)} \geq \lambda_{1}
$$

for some positive constant $\lambda_{1}$. Also $t=d_{X}\left(x_{0}, g_{t} x_{0}\right)$, and the stretch of $f$ satisfies:

$$
\text { p-stre }(f)(v) \geq \frac{\lambda_{1}}{\lambda \lambda_{0}} \liminf _{t \rightarrow \infty} \frac{d_{\widetilde{T}}\left(\widetilde{t_{0}}, F\left(g_{t}\right) \widetilde{t}_{0}\right)}{d_{X}\left(x_{0}, F\left(g_{t}\right) x_{0}\right)}>0
$$

which is positive since the action of $\Gamma$ on $T$ is geometrically engaging and $t \mapsto$ $F\left(g_{t}\right) x_{0}(t \geq 0)$ is a quasi-ray in $X$.

\section{Exotic actions}

We will present a family of geometrically engaging actions which are not of an algebraic nature (see Theorem 3.3). The construction is a modified version of examples previously constructed by Benveniste in [4].

Let $H$ be a noncompact simply connected simple Lie group and $G$ be a noncompact simple Lie subgroup of $H$ with real rank at least 2 and codimension at least 3. Let $\Lambda$ be a cocompact lattice in $H$ such that $\Gamma=G \cap \Lambda$ is a cocompact lattice in $G$. We further assume that $\Lambda$ is torsion free. Also, throughout this section we will assume that $H$ carries a Riemannian metric which is right $H$-invariant and left $L$-invariant, as in the proof of Theorem 3.3, where $L$ is such that $\operatorname{Ad}(L)$ is a maximal compact subgroup of $\operatorname{Ad}(H)$.

The quotient space $H / \Lambda$ has a foliation given by the induced action of $G$ on its left, and it has one compact orbit $C_{0}=G / \Gamma$. The arguments from [4] prove that $C_{0}$ can be blown up to obtain a smooth manifold $M_{0}$ with boundary $B_{0}$ in such a way that $M_{0}$ is acted upon by $G$ with the natural projection $M_{0} \rightarrow H / \Lambda$ a $G$-equivariant map. We now take two copies $M_{0}^{\prime}, M_{0}^{\prime \prime}$ of $M_{0}$ and identify them along their boundary $B_{0}$. The resulting space $T_{0}$ is a smooth manifold which admits a $G$-action on the left. It is clear that the smooth volume on $H$ induces a 
finite $G$-invariant measure on $T_{0}$. We will consider $T_{0}$ as a Riemannian manifold with metric induced by metrics in $M_{0}^{\prime}$ and $M_{0}^{\prime \prime}$ coming from one on $M_{0}$.

A similar construction, which will prove to simplify certain matters, can be performed without having to blow up before gluing. More specifically, let us take two copies of $H / \Lambda$ and identify them along the orbit $C_{0}=G / \Gamma$ to obtain a space $T_{0}^{\prime}$. The space $T_{0}^{\prime}$ is a manifold with singularities which admits a length structure simply by defining the distance between two points to be the minimum of the lengths of all paths joining them, where the length is measured according to which copy of $H / \Lambda$ the segments of the path lie in. Also observe that there is a natural $G$-equivariant projection $\pi_{0}: T_{0} \rightarrow T_{0}^{\prime}$ induced by the projection $M_{0} \rightarrow H / \Lambda$.

Let us now assume that the centralizer $Z=Z_{H}(G)$ is not discrete and choose $z \notin G \Lambda$. Let $M_{z}$ be the smooth manifold obtained by blowing up the compact $G$-orbits $C_{0}=G / \Gamma$ and $C_{1}=G z / \Gamma$. Again, it is proved in [4] that the left $G^{-}$ action on $H / \Lambda$ induces a left $G$-action on $M_{z}$ such that the natural projection $M_{z} \rightarrow H / \Lambda$ is $G$-equivariant. Denote by $T_{z}$ the manifold obtained from $M_{z}$ by identifying the two exceptional divisors associated to $C_{0}$ and $C_{1}$. Clearly, the left $G$-action on $M_{z}$ induces one on $T_{z}$. In this construction, whenever it is necessary to choose Riemannian metrics we will assume that a metric on $T_{z}$ is fixed and induces one on $M_{z}$.

Once again, we perform the construction without blowing up and denote by $T_{z}^{\prime}$ the space obtained from $H / \Lambda$ by identifying $C_{0}$ with $C_{1}$. The resulting space is a manifold with singularities carrying a length structure as the one described for $T_{0}^{\prime}$ where the length of a curve is now measured by carrying suitable segments from $T_{z}^{\prime}$ to $H / \Lambda$ and computing their lengths; observe that the right $H$-invariance of the metric on $H$ implies that the length of a segment within the image of $C_{0} \cup C_{1}$ in $T_{z}^{\prime}$ is independent of whether it is computed as a curve in $C_{0}$ or in $C_{1}$. We have again a map $\pi_{z}: T_{z} \rightarrow T_{z}^{\prime}$ which is induced by the projection $M_{z} \rightarrow H / \Lambda$ and hence is $G$-equivariant.

We observe that both universal covers $\widetilde{T}_{0}^{\prime}$ and $\widetilde{T}_{z}^{\prime}$ have length space structures that can be obtained in the same manner as for $T_{0}^{\prime}$ and $T_{z}^{\prime}$. For these metrics the covering maps are clearly local isometries. When required, we will assume that $\widetilde{T}_{0}^{\prime}$ and $\widetilde{T}_{z}^{\prime}$ carry such metrics.

Remark 4.1. Observe that the universal cover $\widetilde{T}_{0}$ of $T_{0}$ can be constructed by hand and for our purposes all we need to know about the construction is the following. One takes the universal cover $\widetilde{M}_{0}$ of $M_{0}$, which can be seen as $H$ with the translates $G \lambda$ of $G(\lambda \in \Lambda)$ blown up. Then one considers a suitable collection $\left(\widetilde{M}_{i}\right)_{i}$ of copies of $\widetilde{M}_{0}$ and glue one copy $\widetilde{M}_{i}$ to another $\widetilde{M}_{j}$ along some exceptional divisor (i.e. some lift of $B_{0}$ ) which is not necessarily the same within $\widetilde{M}_{i}$ and $\widetilde{M}_{j}$. The manner in which the gluing is to be performed is dictated by the structure of the fundamental group of $T_{0}$, which is $\Lambda *_{\Gamma} \Lambda$ (the amalgamated product build from the identity isomorphism in $\Gamma$ ), and it is not of importance for the argument. However, one important feature that we will need is that if a 
path joining two points within the same copy $\widetilde{M}_{i}$ moves crossing a submanifold $B$ through which it is glued to a copy $\widetilde{M}_{j}$, then it has to come back to $\widetilde{M}_{i}$ through the same submanifold $B$.

Similar observations apply to the universal covers of $T_{0}^{\prime}, T_{z}$ and $T_{z}^{\prime}$, where we use copies of $H$ for both $\widetilde{T}_{0}^{\prime}$ and $\widetilde{T}_{z}^{\prime}$ suitably identified along the translates $G \lambda$ in the case of $\widetilde{T}_{0}^{\prime}$, and identified along the translates $G \lambda$ and $G z \lambda$ in the case of $\widetilde{T}_{z}^{\prime}$. For the universal cover $\widetilde{T}_{z}$ we use a family of copies of $\widetilde{M}_{z}$. Also observe that the fundamental group of both $T_{z}$ and $T_{z}^{\prime}$ is the HNN-extension build out of $\Gamma \subset \Lambda$, for the isomorphism $\Gamma \rightarrow \Gamma$ given by the identity (see [4] for more details), and which we will denote by $\Lambda *_{\Gamma}$ from now on.

Remark 4.2. An additional important feature to notice about the universal cover $\widetilde{T}_{0}^{\prime}$ of $T_{0}^{\prime}$ is that if a path $\gamma$ has both endpoints in a fixed copy $H_{i}$ of $H$, then one can get a curve $\gamma_{0}$ lying completely within that copy with the same endpoints and length.

To obtain $\gamma_{0}$ we use the previous remark and the following observation. If $\alpha$ is a path lying in a copy $H_{i}$ with endpoints in a translate $G \lambda \subset H_{i}$ glued to $G \lambda^{\prime} \subset H_{j}$ for some other copy $H_{j}$, then there is a path $\alpha^{\prime}$ lying in $H_{j}$ with the same endpoints and length; $\alpha^{\prime}$ is obtained by taking the mirror image of $\alpha$ through $G \lambda$, i.e. by considering in $H_{j}$ the path that corresponds to $\alpha$ under the natural isometry $H_{i} \cong H \cong H_{j}$ composed with the isometry given by right multiplication by $\lambda^{-1} \lambda^{\prime}$. Then $\gamma_{0}$ is obtained from $\gamma$ by repeatedly taking the mirror images of subarcs of $\gamma$. In particular, for any two points $x$ and $y$ lying in the same copy $H_{i}$ of $H$ in $\widetilde{T}_{0}^{\prime}$, we have:

$$
d_{\widetilde{T}_{0}^{\prime}}(x, y)=d_{H_{i}}(x, y)
$$

where both $d_{\widetilde{T}_{0}^{\prime}}$ and $d_{H_{i}}$ are length metrics, i.e. the infimum of the length of the paths joining two points within the corresponding space.

A similar remark applies to the universal cover $\widetilde{T}_{z}^{\prime}$ of $T_{z}^{\prime}$. Here we only need to notice that besides translating with a right multiplication by $\lambda^{-1} \lambda^{\prime}$ we may first have to translate with the right multiplication by $z$, which is still an isometry.

Lemma 4.3. With a setup as above, for fixed Riemannian metrics on $T_{0}$ and $T_{z}$, there is a constant $A>0$ such that for any choice of lifts $\widetilde{\pi}_{0}$ and $\widetilde{\pi}_{z}$ to universal covers of the maps $\pi_{0}$ and $\pi_{z}$, respectively, we have:

$$
\begin{aligned}
& d_{\widetilde{T}_{0}^{\prime}}\left(\widetilde{\pi}_{0}(x), \widetilde{\pi}_{0}(y)\right) \leq A d_{\widetilde{T}_{0}}(x, y) \quad \forall x, y \in \widetilde{T}_{0} \\
& d_{\widetilde{T}_{z}^{\prime}}\left(\widetilde{\pi}_{z}(x), \widetilde{\pi}_{z}(y)\right) \leq A d_{\widetilde{T}_{z}}(x, y) \quad \forall x, y \in \widetilde{T}_{z}
\end{aligned}
$$

where the distances in the universal covers $\widetilde{T}_{0}^{\prime}$ and $\widetilde{T}_{z}^{\prime}$ come from the length structures described before. 
Proof. The natural projection $M_{0} \rightarrow H / \Lambda$ is smooth and has compact domain. Therefore, there is a constant $A>0$ such that for any curve $\alpha$ in $\widetilde{M}_{0}$ with image $\alpha^{\prime}$ in $H$ under a lift of $M_{0} \rightarrow H / \Lambda$ we have:

$$
\operatorname{length}_{H}\left(\alpha^{\prime}\right) \leq A \operatorname{length}_{\widetilde{M}_{0}}(\alpha)
$$

In view of the remarks preceding this lemma, any curve $\alpha$ in $\widetilde{T}_{0}$ can be decomposed into segments lying within a copy of $\widetilde{M}_{0}$. And for any such segment the previous inequality applies, so that after adding up the length of all segments we have:

$$
\text { length }_{\widetilde{T}_{0}^{\prime}}\left(\alpha^{\prime}\right) \leq A \text { length }_{\widetilde{T}_{0}}(\alpha)
$$

and from this we obtain the first inequality in our statement. The second inequality is proved similarly.

As for geometrical engagement for the exotic actions we have the following:

Theorem 4.4. For $G, T_{0}$ and $T_{z}$ as above, the actions of $G$ on $T_{0}$ and $T_{z}$ are geometrically engaging.

Proof. First observe that from the previous Lemma (since we can assume that the mappings $\widetilde{\pi}_{0}$ and $\widetilde{\pi}_{z}$ are $G$-equivariant) it is enough to check that the limit inferior condition from Definition 2.7 is satisfied for the length spaces $\widetilde{T}_{0}^{\prime}$ and $\widetilde{T}_{z}^{\prime}$. But the latter is a direct consequence of Remark 4.2 and Theorem 3.3.

An inmediate consequence of Theorems $\mathrm{C}$ and 4.4 is the following:

Corollary 4.5. Let $N$ be a compact manifold with nonpositive sectional curvature. If $\pi_{1}(N)$ is isomorphic to the amalgamated product $\Lambda *_{\Gamma} \Lambda$ or to the $H N N-$ extension $\Lambda *_{\Gamma}$ (both constructed from the identity isomorphism of $\Gamma$ ), then there is an isometric totally geodesic immersion of the symmetric space associated to $G$ in $N$. In particular, the fundamental group of a compact manifold with strictly negative sectional curvature cannot be isomorphic to either $\Lambda *_{\Gamma} \Lambda$ or $\Lambda *_{\Gamma}$.

Proof. We only have to observe that $\Lambda *_{\Gamma} \Lambda$ and $\Lambda *_{\Gamma}$ are the fundamental groups of $T_{0}$ and $T_{z}$, respectively.

Examples of Lie groups $G$ and $H$ to which the arguments above can be applied include the case of $G=S L(n) \subset H=S L(n+2)$.

Also, as the reader would probably have noticed, it makes sense to somewhat generalize this construction of amalgamated actions to include the case of a graph of homogeneous spaces. That is, one has a graph $T$, associated to each of its vertices there is a homogeneous space of the form $H / \Lambda$, and associated to each 
edge there is a homogeneous space $G / \Gamma$. Moreover, each edge group come equipped with inclusions on the vertex groups of that particular edge. After that one can form a stratified space by appropriately gluing vertex spaces along their edges. The cases that we have considered here are those in which the graph is either an edge, with one or with two vertices, and the vertex spaces are the same. We have not discussed the more general construction because it goes out of the philosophy of the topic, namely, consideration of smooth actions on smooth manifolds. In the general case of a graph, the final amalgamated space is not likely to be a manifold but a stratified space whose strata are homogeneous spaces.

As to whether the techniques of this paper apply to these more general stratified spaces, we note that most of them extend with minor modifications. An exception is Proposition 3.5 which can nonetheless be done in this generalized setup after some more careful consideration. Also, the theory of harmonic maps works pretty well in this setting, as many authors have already shown (e.g. Gromov-Schoen); in particular, the proof of our Proposition 2.15 as found in [8] can be carried out with almost no modification to the case of compact metric spaces with smooth laminations.

\section{Proof of the main theorems}

Theorem A. Let $M$ be a manifold with a smooth foliation $\mathcal{F}$ carrying a finite invariant transverse measure $\mu$ and a leafwise Riemannian metric g. Assume that each leaf is isometrically covered by a fixed irreducible symmetric space $X$ of noncompact type which is either of rank at least 2 or a quaternionic or Cayley hyperbolic space, and that $\operatorname{stre}(M, \mathcal{F}, g)>0$. If $N$ is a compact manifold with nonpositive sectional curvature when $\operatorname{rank}(X) \geq 2$ and with nonpositive complexified sectional curvature otherwise and $\pi_{1}(M) \cong \pi_{1}(N)$, then there exist a homothetic totally geodesic immersion $X \rightarrow N$.

Proof. Since $N$ has contractible universal cover, there is a smooth map $f: M \rightarrow N$ which induces an isomorphism on fundamental groups. Then by Corollary 3.2, the map $f$ has positive stretch, and so by Proposition 2.15 the conclusion follows.

Theorem B. Let $(M, g)$ be a compact Riemannian manifold whose universal cover can be isometrically split as $\widetilde{M} \cong Y \times X$, where $X$ is as in Theorem A. Let $N$ be a compact manifold with nonpositive sectional curvature when $\operatorname{rank}(X) \geq 2$ and with nonpositive complexified sectional curvature otherwise. If $\pi_{1}(M) \cong \pi_{1}(N)$, then there is a homothetic totally geodesic immersion $X \rightarrow N$. In particular, for $\operatorname{rank}(X) \geq 2$ the group $\pi_{1}(M)$ cannot be isomorphic to the fundamental group of a compact manifold with strictly negative sectional curvature.

Proof. By passing to a finite cover of $M$, as well as the corresponding covering of 
$N$ under some isomorphism $\pi_{1}(M) \cong \pi_{1}(N)$, we can assume that the factor $X$ from $\widetilde{M}$ induces a foliation $\mathcal{F}$ on $M$. Moreover, the Riemannian measure on $Y$ induces a finite invariant transverse measure for $\mathcal{F}$. By Proposition 2.16 it follows that $(M, \mathcal{F})$ has positive stretch, and by Theorem A the conclusion follows.

Theorem B'. Let $(M, h)$ be a compact Riemannian manifold without conjugate points carrying a smooth foliation $\mathcal{F}$ which is Riemannian for $h$ with totally geodesic leaves. Assume that the lift of $\mathcal{F}$ to $\widetilde{M}$ has leaves isometric to $X$ as in Theorem $A$ for the restriction of $h$ to the leaves. Let $N$ be a compact Riemannian manifold with nonpositive sectional curvature when $\operatorname{rank}(X) \geq 2$ and with nonpositive complexified sectional curvature otherwise. If $\pi_{1}(M) \cong \pi_{1}(N)$, then there is a homothetic totally geodesic immersion $X \rightarrow N$. In particular, for $\operatorname{rank}(X) \geq 2$ the group $\pi_{1}(M)$ cannot be isomorphic to the fundamental group of a compact manifold with strictly negative sectional curvature.

Proof. The Riemannian transverse structure on $M$ induces a finite invariant transverse measure for $\mathcal{F}$, and since the leaves are totally geodesic in a manifold without conjugate points the pair $(M, \mathcal{F})$ has positive stretch. By Theorem A the conclusion follows.

Theorem C. Let $X$ and $N$ be as in Theorem A, and let $\Gamma$ be a torsion free cocompact lattice of the group of isometries of $X$. Assume $\Gamma$ has a geometrically engaging action on a compact manifold $T$ that preserves a finite measure. If $\pi_{1}(T) \cong \pi_{1}(N)$, then there is an isometric totally geodesic immersion $X \rightarrow N$. In particular, when $\operatorname{rank}(X) \geq 2$, the space $T$ cannot have the fundamental group of a compact manifold with strictly negative sectional curvature.

Proof. By Proposition 3.5 there is smooth map $f: \Gamma \backslash(X \times T) \rightarrow N$ with positive stretch. Hence by Proposition 2.15 the result follows.

\section{References}

[1] S. Adams and L. Hernandez, A foliated metric rigidity theorem for higher rank irreducible symmetric spaces, Geom. Funct. Anal. 4 (1994), 483-521.

[2] W. Ballmann and P. Eberlein, Fundamental groups of manifolds of nonpositive curvature, J. Diff. Geo. 25 (1987), 1-22.

[3] W. Ballmann, M. Gromov and V. Schroeder, Manifolds of nonpositive curvature, Birkhäuser, Boston-Basel-Stuttgart, 1985.

[4] J. Benveniste, Rigidity and deformations of lattice actions preserving geometric structures, $\mathrm{Ph}$. D. Thesis, The University of Chicago (1996).

[5] M. Gromov, Foliated Plateau problem, part II: Harmonic maps of foliations, Geom. Funct. Anal. 1 (1991), 253-320.

[6] S. Helgason, Differential geometry Lie groups and symmetric spaces, Pure and Applied Mathematics 80, Academic Press, New York-San Francisco-London, 1978.

[7] A. L. Onishchik and E. B. Vinberg, Lie groups and algebraic groups, Springer-Verlag, 
Berlin-Heidelberg, 1990.

[8] R. Quiroga-Barranco, The stretch of a foliation and geometric superrigidity, Trans. Amer. Math. Soc. 349 (1997), 2391-2426.

[9] R. J. Spatzier and R. J. Zimmer, Fundamental groups of negatively curved manifolds and actions of semisimple groups, Topology 30 (1991), 591-601.

A. Candel

Department of Mathematics

California Institute of Technology

Pasadena, CA 91125

USA

(Received: October 22, 1997)
R. Quiroga-Barranco

Departamento de Matemáticas

CINVESTAV-IPN

Apartado Postal 14-740

México DF 07300

México

e-mail: quiroga@math.cinvestav.mx 